\title{
Frontières
}

\section{"Mourir avant de mourir » afin de créer sa propre éternité}

\section{Mojgan Mahdavi Zadeh}

Volume 19, numéro 2, printemps 2007

Penser sa mort ?

URI : https://id.erudit.org/iderudit/017501ar

DOI : https://doi.org/10.7202/017501ar

Aller au sommaire du numéro

Éditeur(s)

Université du Québec à Montréal

ISSN

1180-3479 (imprimé)

1916-0976 (numérique)

Découvrir la revue

Citer cet article

Mahdavi Zadeh, M. (2007). « Mourir avant de mourir » afin de créer sa propre éternité. Frontières, 19(2), 68-71. https://doi.org/10.7202/017501ar d'utilisation que vous pouvez consulter en ligne.

https://apropos.erudit.org/fr/usagers/politique-dutilisation/ 


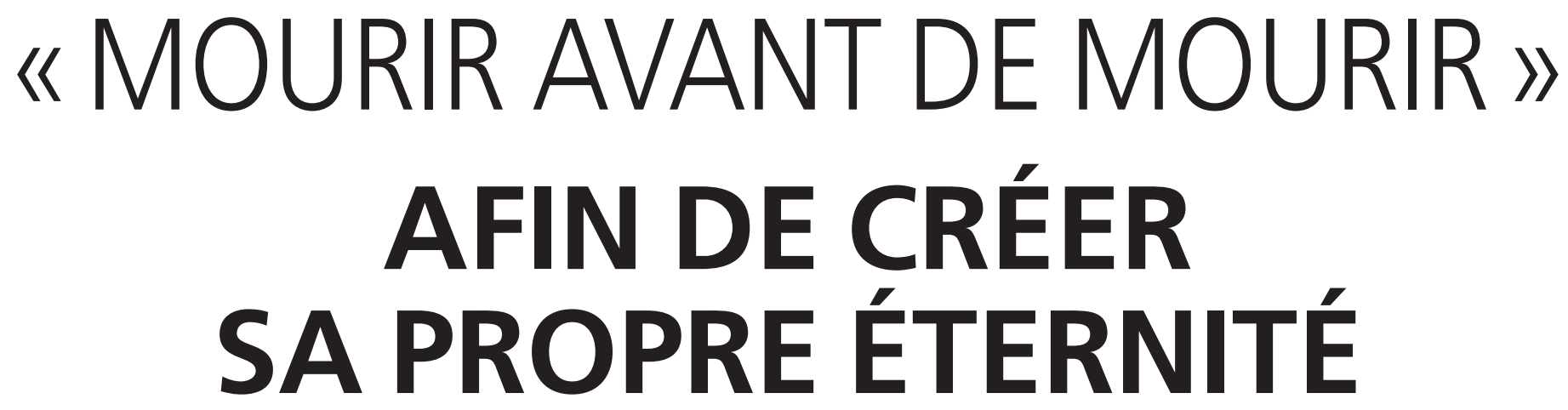

\footnotetext{
Mojgan Mahdavi Zadeh, Ph. D., Département de français, Faculté des langues étrangères, Université d'Ispahan, Iran.
}

Le mysticisme suppose la possibilité d'une communication intime de l'homme avec la divinité par la contemplation et l'extase; l'être humain pourrait ainsi s'élever jusqu'à Dieu et le voir en quelque sorte face à face, sans le secours de la raison. Autrement dit, c'est l'art d'écouter la grande symphonie des sphères célestes, du monde et de son propre être intérieur. Le but du mystique n'est que la vision de Dieu, la contemplation de sa majesté et la compréhension des mystères de la création; c'est entendre l'inouï, voir l'invisible et sentir l'immatériel; c'est voir la richesse incroyable du monde en chaque instant et chaque lieu, sentir la présence divine partout.

Mawlânâ Djalâl-Od-Dîn Rûmî (12071273 ) est un grand mystique d'origine persane pour qui l'amour est une grâce, un feu et une ivresse. Après avoir rencontré un derviche qui s'appelait Chams de Tabriz, il en est tombé amoureux. Ils se sont enfermés pendant quarante jours et quarante nuits. Depuis, Rûmî, n'était plus Rûmî; il dansait en tournant, il chantait, il disait des poèmes, il riait... Chams transforma Rûmî et l'amena à faire l'expérience de la cognition directe. Le Mathnavî, ouvrage monumental en six volumes d'enseignement spirituel rédigé par Rûmî, constitue l'un des trésors de la littérature persane. Rûmî considère que tout individu, depuis les débuts de son éveil mental, est en quête de sens. Le psychisme d'un être humain a pour lui autant d'intérêt que ses caractères moraux. Il a su déchiffrer les intentions de l'homme et nous a présenté le trajet à parcourir pour être celui ou celle que nous devons être. Rûmî se fonde sur une psychologie transcendantale distinguant en l'homme des degrés à gravir pour atteindre la dimension d'intériorité qui lui révélera son moi véritable. Selon une constante loi d'analogie, les états subjectifs ont leur correspondance dans l'échelle universelle de l'Être, qui s'élève du minéral à l'ange, et au-delà. Pour Rûmî, «le monde entier est comme un miroir de l'Unicité divine, qui y apparaît réfractée dans la diversité des phénomènes. En se trouvant lui-même, l'homme retrouve l'univers » (Rûmî, 2003, p. 237).

La doctrine de Rûmî est fondée sur l'amour et la tolérance. Il nous a appris qu'il faut donner son cœur au Créateur, parler toujours d'amour et aimer toutes les créatures puisqu'elles font partie de leur Créateur. Mais comme le vaste océan ne peut remplir un vase au-delà de sa capacité, notre perception des paroles du Mowlâna (l'Amoureux) sera elle aussi proportionnelle à notre capacité de les entendre (De Vitray Meyerovitch et Mokri, 1973, vers 581). Rûmî nous apprend que l'univers entier est sous le commandement de l'homme qui s'est purifié le cœur et l'esprit de toutes les impuretés et qui a touché à l'amour et l'unicité divine. Dans son chef-d'œuvre intitulé Mathnavî Maanavî, il prend la parole et nous demande de bien l'écouter:

Écoute le ney raconter une histoire, il se lamente de la séparation:

Depuis qu'on m'a coupé de la jonchaie, ma plainte fait gémir l'homme et la femme.

Je veux un cœur déchiré par la séparation pour y verser la douleur du désir.

Quiconque demeure loin de sa source aspire à l'instant où il lui sera à nouveau uni.

(Rûmî, 2004, vers 1-4) 
Débarquement des amoureux dans l'île enchantée (1556-1565), Haft Auranf de Djami.

Dans ce poème, Rûmî s'assimile à un roseau (le ney) qui, amoureux de Dieu, s'est vidé totalement de tout «moi » pour être le lieu de la manifestation du souffle divin. Et comme il s'est anéanti dans la voie divine jusqu'au point où il n'a plus rien à dire, c'est son «moi profond» ou plutôt la partie divine de son être qui sera révélée en apportant son souffle à la flûte de roseau de son existence. Depuis, ce n'est plus Mowlâna (l'Amoureux) qui parle mais plutôt Dieu (l'Amant). Et comme l'action de se vider de sa propre personnalité et d'être rempli de celle de son amant n'est point faisable sans amour, c'est à ce momentlà que Mowlâna, lui-même un homme parfait, sera le point de convergence de l'amoureux, de l'amant et de l'amour; là où il parcourra la voie de la remontée vers l'origine et touchera l'unicité divine.

En ce qui concerne l'origine et le retour, il convient de dire que non seulement d'après les traditions islamiques, mais aussi d'après l'Ancien et le Nouveau Testament, les poèmes de Rûmî et bien d'autres poèmes mystiques, l'homme est fait à l'image de Dieu: "Faisons l'homme à notre image comme à notre ressemblance » (Genèse 1, p. 26); "Cependant, vous ne saurez vouloir qu'à moins que Dieu veuille» (Le Coran, sourate 77, verset 30) ; "J'ai créé l'homme à mon image» (Safi-Alichah, 1999, p. 125). Il faut donc mettre de côté les diplopies issues de notre perception des côtés positifs et négatifs des faits, essayer de se perfectionner et de faire apparaître sa nature divine, son humilité, sa splendeur..

\section{PEUR DE LA MORT \\ OU PEUR DE SOI?}

Le vrai mystique n'a point peur de la mort, puisqu'il la considère comme la voie sublime d'une ascension céleste. Rûmî l'affirme: «Ceux qui ont poli leur cœur, ont échappé aux parfums et aux couleurs: ils contemplent sans cesse la Beauté divine à chaque instant» (Mathnavî, Livre premier, vers 3492); et plus loin il ajoute: «La mort, qui effraie tous les hommes, ces gens-là la tournent en dérision. Nul ne remporte la victoire sur leurs cœurs: brise la coquille de l'huître, etnon la perle.» (Mathnavî, Livre premier, vers 3495-3496)

Rûmî, comme bien d'autres mystiques, considère la mort du petit «moi » comme une voie de libération de ce monde matériel. Dans le Livre quatrième, à travers l'histoire du soufi qui méditait dans un jardin, Rûmî raconte qu'en réponse aux amis qui lui proposaient de lever la tête

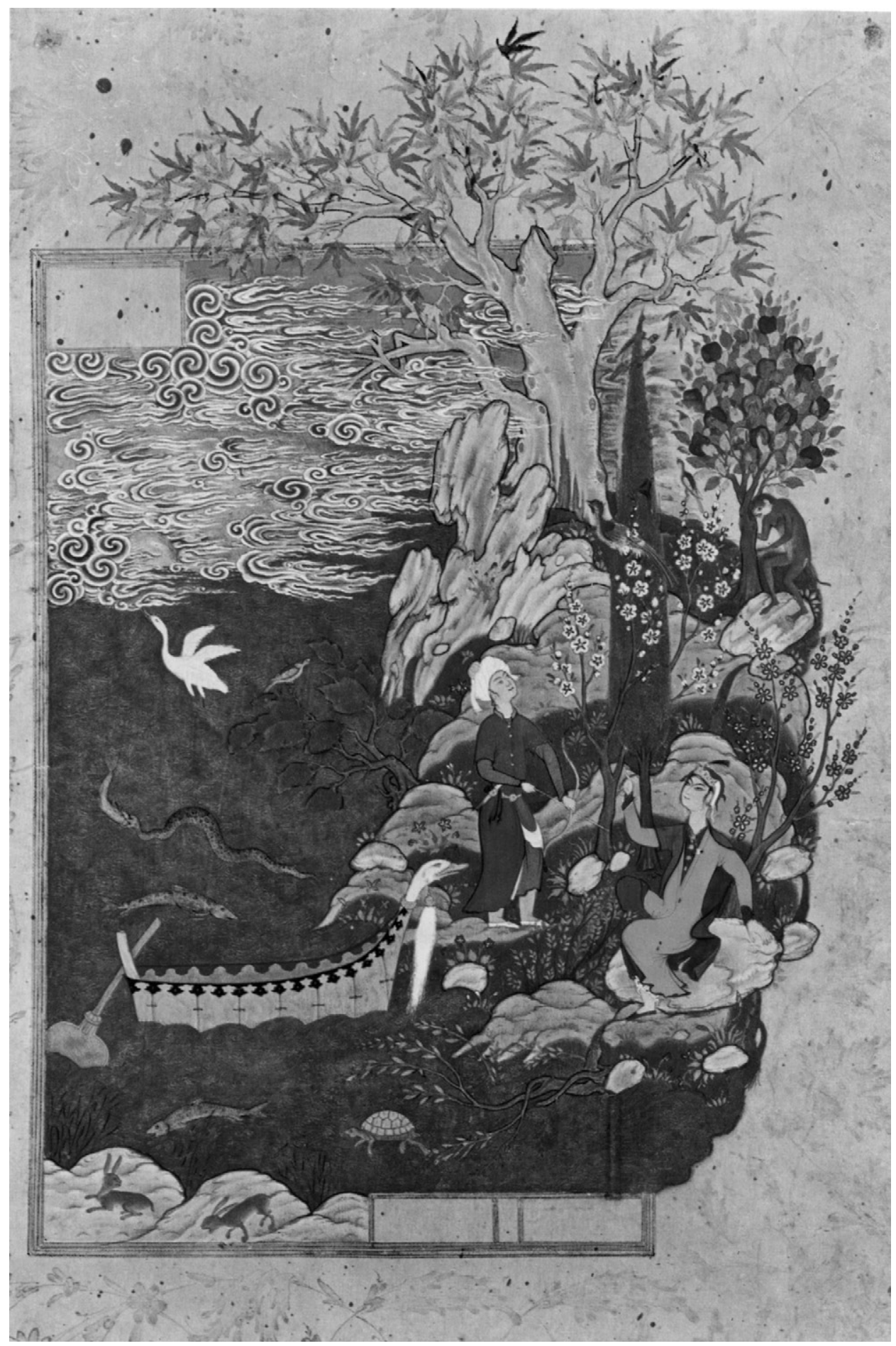

et de se réjouir du jardin et du verger, le soufi répondit: "Ô homme vain, ses signes sont dans le cœur; ce qui est à l'extérieur, n'est que le signe des signes [...] (Mathnavî, Livre quatrième, vers 1362); "Ô, heureux celui qui est mort avant de mourir, car il a perçu le parfum de l'Origine de ce verger. » (Mathnavî, Livre quatrième, vers 1372)

Il y a des moments dans la vie où nous recevons la grâce divine et où nous en sommes tout à fait conscients. Il y a alors un coup de foudre, une étincelle peut-être qui fera flamboyer notre existence. C'est aussi à ce moment que nous connaîtrons le repentir, que nous regretterons tout ce qui nous a fait dévier du vrai chemin. Et quel est-il ce vrai chemin? Eh bien, c'est un chemin qui nous mène à la perfection, exactement comme les gouttes d'eau qui, s'unissant avec l'océan, ne s'appellent plus gouttes d'eau, mais océan. Rûmî l'affirme; il en parle dans son Livre troisième: "Quand l'eau dans l'aiguière va dans l'eau de la rivière, elle disparaît en elle et devient la rivière." (Mathnavî, Livre troisième, vers 3913)

Étant donné que toute chose est périssable (Le Coran, sourate 28, verset 88 ), 
le fait de penser notre propre mort nous afflige, provoque en nous de la panique. Mais Rûmî croit que tout homme qui a peur de la mort a peur de lui-même:

La mort de chacun est de la même qualité que lui-même, mon fils ; pour l'ennemi de Dieu, elle est un ennemi, et pour son ami, un ami.

Aux yeux d'un Turc, le miroir a une couleur claire: de même aux yeux de l'homme noir, le miroir est sombre comme un Noir.

Ta peur de la mort en la fuyant est en réalité la peur de toi-même. Prends garde, ô mon âme!

C'est la laideur de ton propre visage, non le visage de la Mort: ton esprit est comme l'arbre, et la mort comme le feuillage.

(Mathnavî, Livre troisième, vers 3439-3442)

\section{ÉTAPES À FRANCHIR}

Il est tout à fait normal que le chemin à parcourir soit, pour un Occidental, différent de ce qu'il est pour un Oriental; mais étant donné que le but est unique, il existe des réseaux d'associations d'images et d'idéologies qui unifient leur manière de penser. En d'autres termes, un poète mystique dépasse le temps et l'espace. Il n'appartient pas à une époque précise, ni à un lieu spécifique. C'est d'ailleurs ce qui permettrait de s'ouvrir à un horizon d'envergure internationale.

Pour Rûmî, cette façon de mourir avant de mourir comprend plusieurs étapes. La première étape à franchir afin de parvenir à l'annihilation de son propre moi est d'être conscient de la possibilité d'en arriver à une telle mort mystique. Dans son Livre sixième, Rûmî nous demande de nous réveiller de notre lourd sommeil: "Alors, ô dormeurs, gémissez pour vousmêmes, car ce lourd sommeil est une mort mauvaise. » (Mathnavî, Livre sixième, vers 796.) Il faut aussi se libérer de toute sorte de dépendance matérielle, en particulier de l'attachement à notre moi individuel. Cette individualité provient d'une séparation provisoire qui ressemble à un exil. La nostalgie éprouvée à la suite de cette séparation est comparée à celle du roseau coupé de sa jonchaie originelle: "Je l'ai éprouvé : ma mort est dans la vie. Quand j'échapperai à cette vie, ce sera pour durer à jamais. Tuez-moi, tuez-moi, ô mes amis! Car dans ma mort, il y a vie sur vie»(Mathnavî, Livre troisième, vers 3838-3839). Il faut savoir comment combattre notre moi. Il est évident que même les mystiques les plus accomplis n'échappent pas à ce combat: ils sont parfois totalement vidés de tout «moi », pour être le lieu où se manifeste le souffle divin, et parfois, ils se laissent tromper par ce «moi» qui perdure et qui cherche à les faire dévier du chemin de la Vérité. L'important, c'est de pouvoir résister. Qu'est-il, ce «moi charnel», sinon une impureté qui recouvre la surface du miroir de notre monde intérieur et qui nous fait tomber dans le monde des ténèbres, de la souillure et de la perversité? Dans son Livre troisième, Rûmî annonce: "Quand tu fermes cette bouche-ci, une autre bouche s'ouvre, qui se nourrit des mystères» (Mathnavî, Livre troisième, vers 3747). Il s'agit là d'une transformation radicale:

Sache que la condition nécessaire [pour obtenir cet aliment] est la transformation de la nature sensuelle, car la mort des hommes pervers provient de leur nature perverse.

Lorsqu'il est devenu naturel pour un être humain de manger de l'argile, il devient pâle, au teint souffreteux, maladif et misérable;

Mais quand sa vilaine nature a été transformée, la laideur disparaît de son visage et il brille comme un flambeau.

(Mathnavî, Livre troisième, vers 43-45)

Pour aborder la deuxième étape, il faut avoir le désir de se perfectionner et de se mettre à la recherche de Dieu. Cette quête doit être continue et perpétuelle. Rûmî nous le demande en disant: "Cette délibération a pour but de vous enseigner que vous devez chercher Dieu lentement et sans arrêt. Un petit ruisseau qui se meut continuellement ne devient pas souillé ni nauséabond. » (Mathnavî, Livre troisième, vers 3506-3507.) Mais pourquoi lentement? Rûmî répond qu'il ne faut surtout pas oublier que cette recherche et cette quête demandent de la sincérité. Et il ajoute: «Il n'est pas juste d'exiger d'un loup qu'il effectue la tâche d'un berger; exiger quelque chose là où cela ne convient pas est une négation de la requête. " (Mathnavî, Livre quatrième, vers 1974)

La troisième étape, pour qui veut "mourir avant de mourir», consiste à parcourir le chemin mystique. Ce chemin se divise à son tour en plusieurs étapes: sept selon certains mystiques, dix ou cinq selon d'autres... Ni dans le Coran, ni dans le Mathnavî, on ne trouve de classification exacte de ce cheminement; ce qui est évident, cependant, c'est l'importance $\mathrm{du}$ repentir et $\mathrm{du}$ fait de se réveiller du sommeil pour être présent dans l'instant. «Le soufi est le fils de l'instant, ô mon ami. Ce n'est pas la règle de la Voie que de dire: Demain. » (Mathnavî, Livre premier, vers 133-134.) Rûmî explique que même selon la Tradition, on insiste beaucoup sur l'importance de faire attention à ce temps insaisissable:

Le prophète a dit: En ces jours, les souffles de Dieu l'emportent: gardez vos oreilles et votre esprit attentifs à ces influences spirituelles. Captez de tels souffles.

Le souffle divin est venu, t'a aperçu et il est parti.

Un autre souffle est arrivé. Prends garde de ne pas manquer celui-là aussi, ô mon ami!

(Mathnavî, Livre premier, vers 1951-1954)

Le but essentiel de la voie proposée par Rûmî est de vaincre notre âme charnelle (le Nafs), qui se manifeste dans l'orgueil ou l'arrogance, la jalousie, le fait de mentir aux autres et à soi-même, la concupiscence ou le fait de se livrer à la débauche, l'avidité, la colère et enfin la dépendance. Pour pouvoir dominer tout cela, il faut vaincre ce petit «moi ». Rumî l'affirme: «Il n'y a pas d'admission dans la salle d'audience de la Majesté divine pour quiconque, s'il n'est mort à lui-même. Quel est le moyen de l'ascension vers le ciel ? Cette non-existence. La non-existence est la foi et la religion des amoureux de Dieu. » (Mathnavî, Livre quatrième, vers 232-233.) La calamité, pour qui s'engage dans cette Voie, est le fait de se croire parfait. Dans son Livre premier, Rumî insiste là-dessus: «Il n'est de pire maladie en ton âme, ô orgueilleux, que la prétention à la perfection.» (Mathnavî, Livre premier, vers 3214-3216)

En ce qui concerne la concupiscence, ou le fait de se livrer à la débauche, Rûmî signale que la nourriture de l'âme charnelle ou du Nafs, est cette concupiscence; c'est grâce à cette nourriture que le Nafs trouve l'occasion de se développer:

Mais parce que la disposition mauvaise est devenue forte, la fourmi du désir est devenue, par l'habitude, un serpent; 
Tue le serpent du désir dès

le commencement; autrement, vois-tu, ton serpent devient un dragon.

(Mathnavî, Livre deuxième,

vers 3471-3472)

Pour conclure, disons que "mourir avant de mourir» est en fait la transformation de ce moi charnel - qui est fasciné par le monde matériel - en un «moi » divin et éternel. De même qu'il existe deux catégories de mort, pour Rûmî il existe aussi deux sortes de naissances; l'une étant le jour où nous sommes venus au monde par le processus naturel de l'accouchement et l'autre, le jour où nous avons pris conscience de notre véritable existence. Pour cette deuxième naissance, c'est-à-dire pour pouvoir nous détacher de ce monde et le prendre pour un lieu transitoire, il faut un certain effort de la volonté. Et c'est justement en ce sens que les hommes diffèrent les uns des autres. Rûmî précise:

Bien que l'œuf du serpent ressemble à l'œuf du moineau en apparence, la distance entre eux est grande.

Ou encore, bien que le pépin du coing ressemble à l'œuf du moineau en apparence, la distance entre eux est grande. $[. .$.

Les feuilles, c'est-à-dire les corps, sont analogues, mais chaque âme vit d'une façon différente.

Dans le bazar, les gens circulent, tous semblables, mais l'un est joyeux, et l'autre attristé.

Il en va de même dans la mort: nous allons tous pareils, mais la moitié d'entre nous sont des perdants, et l'autre moitié heureux comme des empereurs.

(Mathnavî, Livre troisième, vers 3511-3516)

Quelques vers de Rumî pourraient résumer toute cette démarche:

Notre mort, c'est nos noces avec l'éternité. [...]

Pour celui qui est vivant dans la Lumière de Dieu,

La mort de cette âme charnelle est un bienfait. [...]

Attache tes regards sur Dieu, et ne parle pas de ce qui est invisible,

Afin que dans ton regard, il mette un autre regard.

(Rûmî, 1973, Ôdes mystiques, p. 318)

Autrement dit, un amour spirituel doit se manifester vis-à-vis de tout être, de tout objet existant. Chacun alors se sentira attiré et engagé à vivre cet amour que décrit Rûmî. Du point de vue de la créature, l'univers est multiple, mais selon les règles de l'Unicité divine, la Création est une; autrement dit, l'univers est comme un seul être. L'homme ordinaire ne perçoit que la multiplicité de l'univers, tandis que le saint ou le mystique, lorsque sa réalité intérieure s'identifie à celle de la totalité de l'univers, le perçoit dans son unicité; exactement comme les flocons de neige qui nous semblent tous pareils même si nous savons que, lorsqu'ils sont vus au microscope, ils ne se ressemblent pas.

Les paroles divines de Bouddha, de Zoroastre, de Moïse, de Jésus, de Mohammad et des autres saints, mystiques ou prophètes ainsi que les livres sacrés tel le Coran, l'Ancien et le Nouveau Testament, les Védas, l'Avesta, la Kabbale, le Talmud, sont tous d'une grande valeur et sont considérés comme des références dans de nombreuses recherches. Il faut donc considérer la diversité des peuples et des religions, ainsi que la théorie du pluralisme religieux comme une expression de la sagesse tout en estimant la souveraineté de la tolérance. Il faut donc dépasser les barrières dogmatiques, afin de mieux réaliser l'essence universaliste du message divin. Très ouvert aux autres confessions, Rûmî comparait les voies menant à Dieu aux chemins qui convergent tous vers La Mecque. Lors de ses funérailles, tous les habitants étaient là, les musulmans, mais aussi les chrétiens et les Juifs car tous se reconnaissaient en lui : les Juifs avançaient dans le cortège en chantant des psaumes, les chrétiens en proclamant l'Évangile et nul ne songeait à les écarter. Le sultan fit venir les responsables des communautés juive et chrétienne et leur demanda pourquoi ils honoraient ainsi un musulman. Ils répondirent: «En le voyant, nous avons compris la vraie nature de Jésus, de Moïse et de tous les prophètes" (De VitrayMeyerovitch, 1995, p. 97-98).

\section{Bibliographie}

LE CORAN (1991). Traduction intégrale et notes de M. HAMIDULLAH, Qum, Ansariyan Publications.

DE VITRAY-MEYEROVITCH, E. (1995). Islam, l'autre visage, Paris, Albin Michel.

LAROUSSE (1997). Dictionnaire de la langue française, Paris, Larousse.

LAZARD, G. (2002). Dictionnaire persanfrançais, Téhéran, Éditions Gazal.

RÛMÎ, DJALÂL-OD-DÎN (2004). Mathnawî, La quête de l'absolu, traduction d'E. DE VITRAY-MEYEROVITCH, Paris, Éditions du Rocher.

RÛMÎ, DJALÂL-OD-DÎN (2003). Minagareh Échgh (L'Émailleur de l'Amour), texte établi par K. ZAMANI, Téhéran, Éditions Nachreh Ney.

RÛMÎ, DJALÂL-OD-DÎN (2000). Le Mathnavî, texte établi par M. ÉSTÉLAMI, sept volumes, Téhéran, Éditions Méharate.

RÛMÎ, DJALÂL-OD-DÎN (1999). Le Mathnavî Maanavî, texte établi par K. ZAMANI, sept volumes, Téhéran, Éditions Étélââte

RÛMÎ, DJALÂL-OD-DÎN (1973). Ôdes mystiques, Dîvân-é Shams-é Tabrîzî, traduction d'E. DE VITRAY-MEYEROVITCH et M. MOKRI, Paris, Seuil et UNESCO.

SAFI-ALICHAH, M.H. (1999). ErfanOl-Hagh, texte établi par A. ENTEZAM et E. ESTAKHRI, Téhéran, Éditions SafiAlichah.

SAJÂDÎ, S.J., (2004). Dictionnaire des termes et expressions mystiques, Téhéran, Éditions Tahouri. 